\title{
On 2-Round Secure Multiparty Computation
}

\author{
Rosario Gennaro $^{1}$, Yuval Ishai ${ }^{2, \star}$, Eyal Kushilevitz ${ }^{3, \star \star}$, and Tal Rabin ${ }^{1}$ \\ 1 IBM T.J. Watson Research Center \\ \{rosario, talr\}@watson.ibm.com \\ 2 Princeton University \\ yishai@cs.princeton.edu \\ 3 Technion, Israel \\ eyalk@cs.technion.ac.il
}

\begin{abstract}
Substantial efforts have been spent on characterizing the round complexity of various cryptographic tasks. In this work we study the round complexity of secure multiparty computation in the presence of an active (Byzantine) adversary, assuming the availability of secure point-to-point channels and a broadcast primitive. It was recently shown that in this setting three rounds are sufficient for arbitrary secure computation tasks, with a linear security threshold, and two rounds are sufficient for certain nontrivial tasks. This leaves open the question whether every function can be securely computed in two rounds.

We show that the answer to this question is "no": even some very simple functions do not admit secure 2-round protocols (independently of their communication and time complexity) and thus 3 is the exact round complexity of general secure multiparty computation. Yet, we also present some positive results by identifying a useful class of functions which can be securely computed in two rounds. Our results apply both to the information-theoretic and to the computational notions of security.
\end{abstract}

Keywords: Secure multiparty computation, round complexity, lower bounds.

\section{Introduction}

The race for improving the round complexity of cryptographic protocols appears, quite miraculously, to never lose its stean 1 . In this work we study the round complexity of secure multiparty computation. Following the initial plausibility results in this area [32 21:510], considerable efforts have been spent on obtaining round-

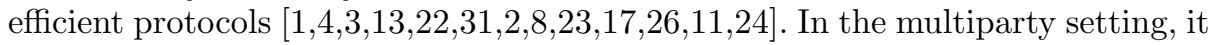
was recently shown in 17 that every function can be securely computed in three rounds (tolerating a constant fraction of malicious players)2 2 and that for certain

* Most of this work was done while the author was at AT\&T Labs-Research.

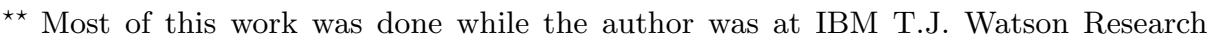
Center.

1 It is somewhat reassuring to note in this context that the round complexity is restricted to be a positive integer.

2 This is possible either with unconditional security, efficiently in the branching program size of the function being computed, or with computational security, efficiently in its circuit size. 
nontrivial tasks two rounds suffice. This naturally raises the question whether every function can be securely computed in two rounds. In the current work we focus on this question, examining the capabilities and limitations of 2-round protocols.

The Model. We consider a system of $n$ players, who interact in synchronous rounds via authenticated secure point-to-point channels and a broadcast medium 3 . Interfering with the interaction is an active (Byzantine) adversary, who may corrupt up to $t$ players (where $t$ is referred to as the security threshold), learn their internal data, and arbitrarily modify their behavior. By default, we make the standard assumption that the adversary has a rushing capability, namely in each round it may learn the messages sent at this round by uncorrupted players to the corrupted players before sending its own messages. This is the most commonly used model in the general secure multiparty computation literature (e.g., [21/51030|28|12]), and in particular it is the standard model assumed in the context of constant-round secure multiparty computation

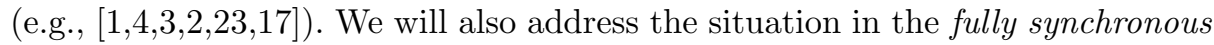
setting, where the messages of each round are guaranteed to be simultaneous. As for other aspects of the model, such as perfect vs. computational security, and adaptive vs. non-adaptive adversary, they can be set appropriately so as to achieve the strongest statements for both our positive and negative results. Section 2 includes a more detailed description of the model and the standard definition of security in this model.

\subsection{Our Results}

We obtain both positive and negative results, resolving the main qualitative questions concerning the possibility of 2-round secure multiparty computation. Our main results are outlined below.

Positive results. We show that any function whose outputs are determined by the input of a single player can be securely computed in two rounds with a linear security threshold, efficiently in its circuit size. In contrast to its appearance, this function class is neither trivial nor useless. In particular, this result generalizes the 2-round protocols for verifiable secret-sharing and secure multicast from [17, implies 2-round distributed zero-knowledge protocols for NP [16, and also has other applications that we discuss. In addition, we observe that there are also functions outside this class which admit 2-round protocols.

Negative results. Our main conclusion is that 3 rounds are necessary for general secure multiparty computation in any "standard" model (i.e., without

\footnotetext{
${ }^{3}$ Broadcast allows each player to send an identical message to all players, without allowing it to violate the consistency requirement. This primitive can be simulated using secure point-to-point channels via a Byzantine Agreement protocol [29|25.7]14]; however the cost of such a simulation would exceed the round complexity we consider here. From a more practical point of view, a broadcast medium may be implemented either physically or via semi-trusted external parties, such as Internet bulletin boards.
} 
public-key infrastructure or preprocessing and with full fairness requirement; see below). Specifically, if $t \geq 2$ (i.e., at least two players may be corrupted), then even some very simple functions cannot be securely computed in two rounds, regardless of the number of players, $n$, and the protocol's communication and time complexity. We consider the simple special cases of $\mathrm{XOR}_{2}^{n}$ and $\mathrm{AND}_{2}^{n}$ (exclusiveor and conjunction of two input bits, where all $n$ players should learn the output) and show that both functions do not admit 2-round protocols. Interestingly, these two cases turn out to be quite different from each other, and each proof represents a distinct type of security concern. Indeed, the impossibility result in the former case is inherently linked to the adversary's rushing capability, as $\mathrm{XOR}_{2}^{n}$ (and more generally, any linear function) admits 2-round protocols in the fully synchronous model. In contrast, the second negative result (for the function $\mathrm{AND}_{2}^{n}$ ) applies also to the fully synchronous model. Naturally, the above two special cases generalize to larger classes of functions with "similar" properties. Combining the above negative results with the 3-round upper bound of [17, we have that 3 is the exact round complexity of general secure multiparty computation with a linear security threshold.

Extension to more liberal models. We note that the above negative results can be extended to the common random string model or, more generally, to a setting where a public string from an arbitrary trusted distribution is given to all players. However, the results do not apply if we allow either a preprocessing stage or distribution of correlated random resources by a trusted party prior to the computation. In fact, in these models two rounds are sufficient for securely computing every function with a linear security threshold. For instance, [18 shows that two rounds are sufficient for achieving independence (i.e., a simultaneous broadcast among $n$ players) given a public-key infrastructure. It follows from our results that some underlying infrastructure is indeed necessary, as otherwise the corresponding functionality is impossible to compute securely in two rounds. Our negative results also rely on the fairness requirement of secure computation, and do not apply if the adversary is allowed to "abort" the computation after learning its output.

\section{$1.2 \quad$ Related Work}

Most relevant to the current work are the works on the round complexity of secure multiparty computation, cited above. Among those, the only work to prove lower bounds on the round complexity is [17], where it is proved that perfect VSS and secure multicast with optimal resilience $(t<n / 3)$ require 3 rounds. However, settling for a slightly smaller security threshold $(t<n / 4)$, these tasks require only two rounds. In contrast, our negative results for 2 rounds apply even when the number of players $n$ is arbitrarily larger than $t$, and even for the relaxed notions of statistical and computational security. We stress though that our negative results do not apply to more liberal settings of secure computation. For instance, if the adversary is passive, then two rounds are sufficient for computing any function with $t<n / 3$; this can be achieved either with computational security, efficiently in the circuit size [4, or with perfect security, efficiently in the 
branching program size [24]. In the two-party case, 2-round secure computation is possible either against a passive adversary in the standard model [32[31], or against an active adversary in the common reference string model, assuming that only one player has an output [8].

The round complexity of zero-knowledge protocols has been extensively studied in various settings (e.g., see [20]). However, this line of work is very different from ours both in the type of task being considered (zero-knowledge vs. general secure computation) and, more importantly, in the setting. Indeed, our multiparty setting is more liberal in the sense that it only requires security against limited collusions of players, and in particular allows to assume that a strict majority of the players remain uncorrupted.

Various papers deal with the round complexity of implementing Byzantine Agreement and broadcast using only point-to-point channels (e.g., see [29[14 27]). While these problems can be viewed as secure computation tasks, they are trivialized in our model since we assume broadcast as a primitive.

Finally, the round complexity of collective coin-flipping (which may also be viewed as a secure computation task) has been discussed in the full information model [6]. This model is dual to the Byzantine Agreement one: it allows only broadcast and no secure point-to-point communication. Similarly to our default model, the adversary is allowed rushing. We note that the availability of secure point-to-point channels in our model makes the coin-flipping task more feasible, and thus negative results from the relevant literature do not apply in our context.

Organization. In Section 2 we present the model and definitions. Section 3 includes our positive results, followed by the lower bounds in Section 4

\section{Model and Definitions}

In this section we outline the definition of secure computation, following Canetti's definition approach [9], and highlight some details that are important for our purposes. The following version of the definition is somewhat simplified. In particular, this simplified version considers a protocol as a stand-alone application and does not support any kind of composition; however, our positive results (and obviously the negative results) hold for stronger versions of the definition as well. We refer the reader to [9,19] for more complete definitions.

Communication model. We consider a network of $n$ processors, denoted $P_{1}, \ldots, P_{n}$ and referred to as players. Each pair of players is connected via a private, authenticated point-to-point channel. In addition, all players share a common broadcast channel, which allows a player to send an identical message to all other players. In some sense, the broadcast channel can be viewed as a medium which "commits" the player to a specific value.

Function. A secure computation task is defined by some $n$-party function $f$ : $\left(\{0,1\}^{*}\right)^{n} \rightarrow\left(\{0,1\}^{*}\right)^{n}$, specifying the desired mapping from the players' inputs to their final outputs. While in certain interesting cases the players will have to reach an agreement on a joint output, the definition allows for each player 
to compute its own output. When referring to a single-output function, it is assumed by default that all players output its value. One may also consider randomized functions, which take an additional random input; however, in this work we focus by default on the deterministic case.

Protocol. Initially, each player $P_{i}$ holds an input $x_{i}$, a random input $r_{i}$, and a common security parameter $k$. The players are restricted to (expected) polynomial time in $k$. The protocol proceeds in rounds, where in each round each player $P_{i}$ may send a "private" message to each player $P_{j}$ (including itself) and broadcast a "public" message, to be received by all players. The messages $P_{i}$ sends in each round may depend on all its inputs $\left(x_{i}, r_{i}\right.$ and $\left.k\right)$ and the messages it received in previous rounds. From now on, we assume without loss of generality that each $P_{i}$ sends $x_{i}, r_{i}, k$ to itself in the first round, so that the messages it sends in each subsequent round may be determined from the messages received in previous rounds. We assume that the protocol terminates after a fixed number of rounds, and each player locally outputs some function of the messages it received.

Adversary. We consider an active t-adversary $\mathcal{A}$, where the parameter $t$ is referred to as the security threshold. The adversary is an efficient interactive algorithm, which is initially given the security parameter $k$ and a random input $r$. Based on these, it may choose a set $T$ of at most $t$ players to corrupt5. The adversary then starts interacting with a protocol (either a "real" protocol as above, or an ideal-process protocol to be defined below), where it takes control of all players in $T$. In particular, it can read their inputs, random inputs, and received messages, and it can fully control the messages they send. (In the weaker setting of passive security, the adversary cannot modify the corrupted players' behavior, but only read their information.) We assume by default that the adversary has a rushing capability: at any round it can first wait to hear all messages sent by uncorrupted players to players in $T$, and use these to determine its own messages. However, we also consider the fully synchronous model, in which the messages sent by the adversary in each round are independent of the messages sent by uncorrupted players in the same round. Finally, upon the protocol's termination, $\mathcal{A}$ outputs some function of its entire view.

Security. Informally, a protocol computing $f$ is said to be $t$-secure if whatever a $t$-adversary can "achieve" by attacking the protocol, it could have also achieved (by corrupting the same set of players) in an ideal process in which $f$ is evaluated using a trusted party. To formalize this definition, we have to define what "achieve" means and what the ideal process is. The ideal process for evaluating the function $f$ is a protocol $\pi_{f}$ involving the $n$ players and an additional, incorruptible, trusted party TP. The protocol proceeds as follows: (1) each $P_{i}$ sends

\footnotetext{
${ }^{4}$ It is usually assumed that the adversary is given an "advice" string $a$, or is alternatively modeled by a nonuniform algorithm. In fact, the proofs of our negative results are formulated in this nonuniform setting, but can be modified to apply in the uniform one as well.

${ }^{5}$ This corresponds to the non-adaptive security model; however, all our results apply to the stronger adaptive model as well.
} 
its input $x_{i}$ to TP; (2) TP computes $f$ on the inputs (using its own random input in the randomized case), and sends to each player its corresponding output. Note that when an adversary corrupts the ideal process, it can pick the inputs sent by players in $T$ to TP (possibly, based on their original inputs) and then output an arbitrary function of all its view (including the outputs it received from TP). To formally define security, we capture what the adversary "achieves" by a random variable concatenating the adversary's output together with the outputs and the identities of the uncorrupted players. For a protocol $\pi$, adversary $\mathcal{A}$, input vector $x$, and security parameter $k$, let $\operatorname{exec}_{\pi, A}(k, x)$ denote the above random variable, where the randomness is over the random inputs of the uncorrupted players, the trusted party (if $f$ is randomized), and the adversary. The security of a protocol $\Pi$ (also referred to as a real-life protocol) is defined by comparing the exec variable of the protocol $\Pi$ to that of the ideal process $\pi_{f}$. Formally:

Definition 1. We say that a protocol $\Pi$ t-securely computes $f$ if, for any (reallife) $t$-adversary $\mathcal{A}$, there exists (an ideal-process) $t$-adversary $\mathcal{A}^{\prime}$ such that the distribution ensembles $\operatorname{exec}_{\Pi, \mathcal{A}}(k, x)$ and $\operatorname{exec}_{\pi_{f}, \mathcal{A}^{\prime}}(k, x)$ are indistinguishable. The security is referred to as perfect, statistical, or computational according to the notion of indistinguishability being achieved. For instance, in the computational case it is required that for any family of polynomial-size circuits $\left\{C_{k}\right\}$ there exists some negligible function neg, such that for any $x$,

$$
\left|C_{k}\left(\operatorname{exec}_{\Pi, \mathcal{A}}(k, x)\right)-C_{k}\left(\operatorname{exec}_{\pi_{f}, \mathcal{A}^{\prime}}(k, x)\right)\right| \leq \operatorname{neg}(k) .
$$

An equivalent form of Definition 1 quantifies over all input distributions $X$ rather than specific input vectors $x$, and gives $X$ as an additional input to the distinguisher $C_{k}$. This equivalent form is convenient for proving our negative results.

Intuitive discussion. Definition 1 asserts that for any real-life t-adversary $\mathcal{A}$ attacking the real protocol there is an ideal-process $t$-adversary $\mathcal{A}^{\prime}$ which can "achieve" in the ideal process as much as $\mathcal{A}$ does in the real life. The latter means that the output produced by $\mathcal{A}^{\prime}$ together with the inputs and outputs of uncorrupted players in the ideal process is indistinguishable from the output (wlog, the entire view) of $\mathcal{A}$ concatenated with the inputs and outputs of uncorrupted players in the real protocol. This concatenation captures both privacy and correctness requirements. On the one hand, it guarantees that the view of $\mathcal{A}$ does not allow it to gain more information about inputs and outputs of uncorrupted players than is possible in the ideal process and, on the other hand, it ensures that the inputs and outputs of the uncorrupted players in the real protocol be consistent with some correct computation of $f$ in the ideal process.

Additional intuition regarding the definition, including our general paradigm for proving negative results, is given in Section 4 .

\section{Positive Results}

Which functions are the easiest to compute securely? In this section we obtain a 2-round protocol for every function whose outputs are determined by the input of 
a single player. We stress that this class of secure computation tasks is nontrivial, and in fact it can be used to implement some important tasks such as VSS and distributed zero-knowledge. To see that this class is nontrivial, note that in the multi-output case the protocol has to ensure that all local outputs be consistent with the same input, and at the same time must hide the players' outputs from each other. Moreover, even in the single-output case (i.e., where the same output is learned by all players) it is not enough to let the player holding the input compute and broadcast the global output; indeed, in this naive protocol the players may not be able to efficiently verify that the broadcasted value is consistent with some valid input.

We now show that every function in the above class can be securely computed in 2 rounds with perfect security and a linear security threshold, efficiently in its circuit size. To prove our claim, we reduce the task of securely computing such a function to that of securely computing a related vector of degree- 2 polynomials, and in return show how to compute such a vector in 2 rounds. We start by describing the latter.

Lemma 1. Let $p=\left(p_{1}, \ldots, p_{s}\right)$ be a vector of degree-2 multivariate polynomials in the inputs $x=\left(x_{1}, \ldots, x_{m}\right)$ over a finite field $F$, where $|F|>n 6$. Moreover, suppose that $P_{1}$ holds the entire input vector $x$ and that each player gets some specified subset of the outputs. Then, $p(x)$ admits a perfectly secure 2-round protocol with a linear security threshold in which the communication and time complexity are linear in $s+m$ and polynomial in the number of players.

Proof sketch: The protocol proceeds similarly to the 2-round VSS protocol from [17. Here we outline a somewhat simplified version which does not achieve an optimal security threshold, but suffices for our purposes. For simplicity, assume that $s=1$; the general case is handled by parallel repetition. In the first round, $P_{1}$ uses the bivariate polynomial secret-sharing of [5] to share each of its inputs; that is, it chooses a random bivariate polynomial $F^{l}(y, z)$ over $F$ of degree at most $t$ in each variable under the condition that $F^{l}(0,0)=x_{l}$ for $l=1,2, \ldots, m$. It sends to player $P_{i}$ the polynomials $f_{i}^{l}(y)=F^{l}(y, i)$ and $g_{i}^{l}(z)=F^{l}(i, z)$. In parallel, each pair of players privately exchange random pads. In the second round, each player $P_{i}$ lets its primary share of $x_{l}$ be $s_{i}^{l}=f_{i}^{l}(0)$ (note that if $P_{1}$ is honest then the points $\left(i, s_{i}^{l}\right)$ lie on a degree-t polynomial) and sends, to each player who should receive the output, the value $p\left(s_{i}^{1}, \ldots, s_{i}^{m}\right)$, i.e. its share of the output $p(x)$ 7. In parallel, each player $P_{j}$ broadcasts the value of each secondary share $f_{i}^{l}(j)$ and $g_{i}^{l}(j)$ masked with the pad exchanged with $P_{j}$ in Round 1. These broadcasts induce an inconsistency graph, each edge of which represents a conflict between secondary shares of different players that were supposed to be equal (if $P_{1}$ and the two relevant players were honest).

\footnotetext{
6 This assumption on the size of $F$ can be eliminated by the use of extension fields.

7 To guarantee privacy, these values have to be randomized so that they lie on a random degree-2t polynomial with $p(x)$ as its free coefficient. We ignore this detail, since it complicates the presentation and is addressed in a standard way by letting $P_{1}$ share additional random values.
} 
We now describe how each player reconstructs the value of $p$ from the $n$ output shares it received and from the (public) inconsistency graph. Suppose that $n>6 t$. The players run a deterministic 2-approximation algorithm for vertex cover on the inconsistency graph [15]. If it returns a vertex cover of size $>2 t$ (implying that there is no vertex cover of size $t$ ), then it is clear that $P_{1}$ is dishonest, and the output is taken to be $p(0)$. Otherwise, let $I$ be the complement of the vertex cover (which is an independent set in the graph). Note that $|I|>4 t$, and so the players in $I$ contain at least $3 t+1$ uncorrupted players whose input shares were all consistent with some input vector $x^{\prime}=\left(x_{1}^{\prime}, \ldots, x_{m}^{\prime}\right)$, and thus their output shares lie on a degree-2t polynomial with free coefficient $p\left(x^{\prime}\right)$. The output value is computed from the $|I|$ output shares of the players in $I$ by applying a Reed-Solomon error correction procedure to find the "nearest" degree$2 t$ polynomial, and taking its free coefficient. Note that if $P_{1}$ is uncorrupted then, since the distance of the relevant code is greater than $2 t$, the correct output will be computed. Conversely, if $I$ indeed contains more than $4 t$ players, then the output will be consistent with the value of $p$ on some input $x^{\prime}$ defined by the (consistent) shares of the uncorrupted players in $I$.

Theorem 1. Suppose that $f$ is a deterministic function whose inputs are all held by a single player. Then, $f$ admits a perfectly secure 2-round protocol with a linear security threshold, computing $f$ efficiently in its circuit size.

Proof. We prove the theorem for the case of a single-output function represented by a boolean circuit; a proof for the general case proceeds similarly. We reduce the secure computation of $f$ to the secure computation of degree- 2 polynomials. Suppose that $C$ is a boolean circuit computing $f$, and let $F$ be a finite field where $|F|>n$. We construct a vector $p$ of degree-2 polynomials over $F$. The input variables of $p$ are of three types: (1) variables $x$, such that $x_{i}$ corresponds to the $i$-th input of $f ;(2)$ variables $y$, such that $y_{i}$ corresponds to the $i$-th intermediate wire in $C$ (i.e., excluding input and output wires); (3) variables $z$, such that $z_{i}$ corresponds to the $i$-th output wire of $C$. The vector $p(x, y, z)$ will serve to verify that the input values $x$ are valid (i.e., each of these variables is assigned either 0 or 1 ), and that the wire labels $y, z$ are consistent with the gates of $C$ and the inputs $x$. Specifically, it should hold that $p(x, y, z)$ is the zero vector if all the above consistency requirements are met, and otherwise it contains at least one nonzero entry. Note that each atomic consistency requirement can be verified by a single degree- 2 polynomial. For instance, the validity of an input value $x_{i}$ can be verified by the polynomial $x_{i}\left(1-x_{i}\right)$, and the consistency of an internal NAND gate having input wires $i, j$ and output wire $k$ can be verified by $1-y_{i} y_{j}-y_{k}$. Hence, the total length of $p$ is proportional to the size of $C$.

Now, let $p^{\prime}(x, y, z)=p(x, y, z) \circ z$ (where $\circ$ denotes concatenation). Given a secure protocol $\Pi$ for computing $p^{\prime}(x, y, z)$, a secure protocol for $C(x)$ proceeds as follows:

- On input $x \in\{0,1\}^{m}$, player $P_{1}$ computes the wire labels $y, z$, and invokes $\Pi$ on inputs $x, y, z$. Let $v \circ z$ denote the output of $\Pi$ (by its security, the same output must be obtained by all uncorrupted players). 
- Each player computes its output as follows: If $v=0$ output $z$, otherwise output $C(0)$.

The correctness of this reduction can be sketched as follows. If $P_{1}$ is honest, the correct output $z=C(x)$ will clearly be obtained (even in the presence of an active $t$-adversary), and no additional information about $x$ will be revealed. Conversely, for either of the two possibilities for obtaining the output, it must be consistent with some input $x$.

As a corollary of Theorem 1, it is possible to obtain 2-round distributed zeroknowledge protocols for NP 16]. Indeed, if $R$ is a polynomial-time predicate defining an NP-language, $P_{1}$ can prove that it knows a witness $w$ such that $R(x, w)=1$ by invoking a secure protocol for the function $f(x, y)=x \circ R(x, y)$ (substituting $w$ for $y$ ). Theorem[1 can also be applied for obtaining a wide array of "certified secret-distribution" schemes, generalizing the VSS primitive. For instance, let $D(s, r)$ be a $(t, n)$-secret-sharing scheme (such that $D_{i}(s, r)$ is the share of the secret $s$ held by $\left.P_{i}\right)$, and let $R(s)$ be an efficient predicate testing whether $s$ satisfies some validity condition. Define a function $f$ whose inputs $s, r$ are held by $P_{1}$, and such that $P_{i}$ 's output is $\left(D_{i}(s, r), R(s)\right)$. Then, the secure computation of $f$ allows $P_{1}$ to securely distribute his secret $s$ among $n$ players ensuring consistency of the shares with some valid secret $s$, which can at a later stage be reconstructed even in the presence of faulty players. Note that if $P_{1}$ fails to pick the input $r$ at random, then at most the secrecy of the secret $s$ is compromised (which anyway cannot be avoided) but not its validity.

We end this section by noting that Theorem 1 does not cover all functions which admit 2-round protocols. We demonstrate this using the following "degenerate" example, which in fact requires only one round, but more interesting examples (requiring 2 rounds) can be obtained.

Example 1. Consider the function $f\left(x_{1}, x_{2}, \ldots, x_{n}\right)=\left(x_{1} \oplus x_{2}, \perp, \ldots, \perp\right)$. The value $x_{i}$ is the input of player $P_{i}$, only player $P_{1}$ should output the exclusive-or of the bits $x_{1}, x_{2}$ and other players have no output. Note that the output of $f$ depends on inputs of two players. Yet, it can be verified that the trivial protocol, in which $P_{2}$ sends its input to $P_{1}$ and the latter computes the correct output, is $t$-secure for any threshold $t$.

In the next section we will show that the above function does not admit a 2-round protocol if all players should output $x_{1} \oplus x_{2}$.

\section{Negative Results}

In this section we prove impossibility of 2-round secure computation for some simple specific functions. Since defining the notion of security is a delicate issue, it is not surprising that negative results may also involve some subtleties. In particular, one has to account for all possible strategies of the ideal-process adversary, who is not restricted to any particular behavior pattern. Our general paradigm for proving negative results is the following. For a given function $f$ and 
a protocol $\Pi$, we define a specific real-life adversary $\mathcal{A}_{0}$ which "breaks" $\Pi$ in the sense that it has some advantage over any ideal-process adversary $\mathcal{A}^{\prime}$ attacking $\pi_{f}$. To demonstrate this, we typically define some distribution on the inputs of uncorrupted players, and then specify some concrete "challenge" which no $\mathcal{A}^{\prime}$ can meet (in the ideal process) as successfully as $\mathcal{A}_{0}$ by corrupting the same players as $\mathcal{A}_{0}$ does. For specifying such a challenge, we may use any predicate on the inputs and outputs of uncorrupted players. For instance, $\mathcal{A}_{0}$ may challenge $\mathcal{A}^{\prime}$ to guess the input of a specific uncorrupted player, or to fix the output of some uncorrupted player to 0 . If we show that $\mathcal{A}_{0}$ can significantly outperform every $\mathcal{A}^{\prime}$ in meeting such a challenge, then we have shown $\Pi$ to be insecure.

\subsection{The Functions SB and XOR, or: The Power of Rushing}

In this section, we prove negative results for simultaneous broadcast (defined below) and XOR. A common characteristic of these tasks is that they become easier in the fully synchronous model; thus, in addition to proving the necessity of 3 rounds, these examples also serve to separate the fully synchronous model from the standard model.

We start by showing the impossibility of a 2-round simultaneous broadcast (SB) protocol. This natural task is formally defined by the function $\mathrm{SB}\left(x_{1}, x_{2}\right.$, $\left.\ldots, x_{n}\right)=x_{1} \circ x_{2}$, i.e., each player should output the concatenation of the first two inputs. (We refer to each of the two parts of the global output as an output entry.) Note that the main security requirement imposed by an SB protocol is independence: any ideal-process adversary attacking at most one of the first two players should be unable to induce a non-negligible correlation between the two output entries. We obtain our impossibility result by describing a strategy which allows the adversary to break this independence requirement. The high-level idea is the following. The adversary will corrupt one of the two input holders, say $P_{2}$, and some carefully chosen additional player $P_{j}$, where $j>2$. It will pick its Round 1 messages in such a way that will allow its action in Round 2 to have a non-negligible effect on the second output entry (as seen by uncorrupted players). It will then use its rushing capabilities and the fact that $P_{j}$ was "honest-looking" in Round 1 to first learn the first output entry, and then correlate the second entry with the first one. This will contradict the independence requirement.

We now formalize the above intuition and fill in some missing details. It will be convenient to use the following notation. By $(B, M)$, where $M=\left(M_{1}, \ldots\right.$, $M_{n}$ ), we denote some joint distribution of messages and broadcast sent by $P_{2}$ in Round 1 (where $M_{i}$ is the message sent to $\left.P_{i}\right)$. By $\left(B^{0}, M^{0}\right)$ (resp., $\left(B^{1}, M^{1}\right)$ ) we denote the honest distributions corresponding to the input $x_{2}=0$ (resp., $\left.x_{2}=1\right)$. For a distribution $(B, M)$, let $q_{\sigma}(B, M)$ denote the probability that the protocol's second output entry is equal to 1 , given that: (1) $x_{1}=\sigma$; (2) $P_{2}$ 's Round 1 messages and broadcast are distributed according to $(B, M)$; (3) everyone else follows the protocol (including $P_{2}$ in Round 2). Finally, let $q(B, M)=\left(q_{0}(B, M), q_{1}(B, M)\right)$. All the above distributions and probabilities are parameterized by the security parameter $k$, which will usually be omitted. We start with the following lemma. 
Lemma 2. The distributions ensembles $\left(B^{0}, M_{1}^{0}\right)(k)$ and $\left(B^{1}, M_{1}^{1}\right)(k)$ are computationally indistinguishable.

Proof. Assume towards a contradiction that there is a distinguisher $D$ such that $D$ always outputs 0 or 1 , and $\left|\operatorname{Pr}\left[D\left(B^{0}, M_{1}^{0}, k\right)=1\right]-\operatorname{Pr}\left[D\left(B^{1}, M_{1}^{1}, k\right)=1\right]\right|>$ $k^{-c}$, for some constant $c$ and infinitely many values of $k$. We use $D$ to show that an adversary corrupting $P_{1}$ can break the independence requirement. The adversary's strategy is simple: it waits to hear the broadcast $b$ and message $m$ received from $P_{2}$ in Round 1 , evaluates $D(b, m, k)$, and uses the result as its input. Clearly, the correlation induced by the adversary cannot be emulated in the ideal process (even up to computational indistinguishability).

Theorem 2. There is no 2-round (computationally) secure SB protocol, for any $t \geq 2$ and an arbitrarily large number of players $n \geq 3$.

Proof. Assume towards a contradiction that a 2-round SB protocol is given. Consider the following four pairs of probabilities:

$$
\begin{aligned}
& Q_{1}=q\left(B^{1}, M_{1}^{1}, M_{2}^{1}, \ldots, M_{n}^{1}\right) \\
& Q_{2}=q\left(B^{1}, M_{1}^{1}, 0,0, \ldots, 0\right) \\
& Q_{3}=q\left(B^{0}, M_{1}^{0}, 0,0, \ldots, 0\right) \\
& Q_{4}=q\left(B^{0}, M_{1}^{0}, M_{2}^{0}, \ldots, M_{n}^{0}\right)
\end{aligned}
$$

By the protocol's correctness, we must have $Q_{1} \geq(1-$ neg, $1-$ neg $)$ and $Q_{4} \leq$ (neg, neg), where neg denotes some negligible function in $k$. Moreover, by Lemma 2 the difference between $Q_{2}$ and $Q_{3}$ is negligible. Hence, there is a substantial difference either between $Q_{1}$ and $Q_{2}$ or between $Q_{3}$ and $Q_{4}$. Assume wlog that the difference between the first entries of $Q_{1}$ and $Q_{2}$ (corresponding to the probability $\left.q_{0}\right)$ is large, say, more than $1 / 3$. By a hybrid argument, there exists $i \geq 2$ such that $q_{0}\left(B^{1}, M_{1}^{1}, \ldots, M_{i}^{1}, 0, \ldots, 0\right)-q_{0}\left(B^{1}, M_{1}^{1}, \ldots, M_{i-1}^{1}, 0, \ldots, 0\right)>1 /(3 n)$. It follows that one of the two $q_{0}$ probabilities above must be different by at least $1 /(6 n)$ from one of the two corresponding $q_{1}$ probabilities. Assume, without loss of generality, that

$$
\left|q_{0}\left(B^{1}, M_{1}^{1}, \ldots, M_{i}^{1}, 0, \ldots, 0\right)-q_{1}\left(B^{1}, M_{1}^{1}, \ldots, M_{i-1}^{1}, 0, \ldots, 0\right)\right|>\frac{1}{6 n}
$$

(the other cases are similar).

Now, we need to identify two players to complete two tasks in our attack. Yet, it might be the case that both these tasks can be embodied into a single player. We need an honest looking player $P_{j}$ from whose local view we will compute the correct output, and a second player $P_{i}$ who can toggle the output of the rest of the players in the protocol. The index of player $P_{i}$ is given from Eq. (1). If $i>2$ then this player has acted honestly until now and thus can also be "used" as the player $P_{j}$ from which we extract the output. Otherwise, i.e. if $i=2$, then we set $P_{j}=P_{3}$, as the player whose view we will examine. An adversary corrupting $P_{2}, P_{j}$ can correlate the second output entry with the first as follows. Its Round 1 messages are distributed according to $\left(B^{1}, M_{1}^{1}, M_{2}^{1}, M_{3}^{1}, \ldots, M_{i}^{1}, 0\right.$, 
$\ldots, 0)$. In Round 2, it waits to hear the messages from all uncorrupted players, and then computes the first output entry from the entire view of $P_{j}$. Since $P_{j}$ was honest so far, the protocol's correctness guarantees that it learns the correct output with overwhelming probability 8 . Let $\alpha$ be the value of the first output entry computed by $P_{j}$. The adversary correlates its output with $\alpha$ by letting $P_{i}$, the toggling player, act as follows. If $\alpha=0, P_{i}$ behaves honestly (i.e., uses the original message $M_{i}^{1}$ received from $P_{2}$ in Round 1). Otherwise, it behaves as if this message was set to 0 . It follows from Eq. (11) that the second output entry will be significantly correlated with the first, contradicting the independence requirement.

Note that the SB function admits a trivial 1-round protocol in the fully synchronous model; thus in this case coping with a rushing adversary costs two additional rounds. Another observation is that the requirement $t \geq 2$ is essential. Indeed, the following 5-player protocol computes SB with perfect 1-security in two rounds: (1) Each of $P_{1}, P_{2}$ privately sends its input to each of the remaining 3 players; (2) Each of $P_{3}, P_{4}, P_{5}$ passes the inputs it received to all other players; Each player outputs the majority of the 3 candidates for each input it received in Round 2. It is not hard to verify that the above protocol is a 1-secure SB protocol.

The function XOR. We now turn to the function $\operatorname{XOR}\left(x_{1}, x_{2}, \ldots, x_{n}\right)$ defined as $x_{1} \oplus x_{2}$. We show, by refining the previous arguments for the SB function, that this function as well cannot be securely computed in two rounds.

Theorem 3. There is no 2-round (computationally) secure XOR protocol, for any $t \geq 2$ and an arbitrarily large number of players $n \geq 3$.

Proof. Similarly to the proof of Theorem 2, the adversary corrupts the player $P_{2}$ which, together with an additional player (to be chosen carefully), is used to violate the properties of the alleged protocol. We also follow some of the notations used in the proof of Theorem 2. Specifically, by $(B, M)$ we denote some joint distribution of the broadcast and the private messages (respectively) sent by $P_{2}$ in Round 1 of the protocol. By $\left(B^{b}, M^{b}\right)(b \in\{0,1\})$ we denote the honest distribution corresponding to the input $x_{2}=b$. Consider a scenario where $P_{1}$ chooses its input $x_{1}$, at random. In such a case, in the ideal process, the output is totally random (i.e., each value $\{0,1\}$ is obtained with probability of exactly 0.5 ). On the other hand, we will show a strategy for the adversary to significantly bias the output (towards one of the output values). As in the proof of Theorem 2, let $q(B, M)$ be a pair $\left(p_{0}, p_{1}\right)$ indicating the probability that the output of the function (as seen by the good players) is 1 provided that the input $x_{1}$ is 0 or 1 (respectively) and that the first round messages of $P_{2}$ are distributed as in $(B, M)$. As before, consider the following four pairs:

$$
\begin{aligned}
& Q_{1}=q\left(B^{0}, M_{1}^{0}, M_{2}^{0}, \ldots, M_{n}^{0}\right) \\
& Q_{2}=q\left(B^{0}, M_{1}^{0}, 0, \ldots, 0\right)
\end{aligned}
$$

${ }^{8}$ Note that there is no guarantee that the correct output can be inferred from the view of $P_{2}$, since $P_{2}$ has deviated from the protocol in Round 1 . 


$$
\begin{aligned}
& Q_{3}=q\left(B^{1}, M_{1}^{1}, 0, \ldots, 0\right) \\
& Q_{4}=q\left(B^{1}, M_{1}^{1}, M_{2}^{1}, \ldots, M_{n}^{1}\right)
\end{aligned}
$$

By the protocol's correctness, it follows that $Q_{1}=$ (neg, $1-$ neg) while $Q_{4}=$ $\left(1-\right.$ neg, neg). In addition, similarly to Lemma 2. the distributions $\left(B^{0}, M_{1}^{0}\right)$ and $\left(B^{1}, M_{1}^{1}\right)$ must be indistinguishable (as otherwise $P_{1}$, by using its rushing capability in the first round is able to correlate its input $x_{1}$ with input $x_{2}$ and bias the output; this is impossible to achieve in the ideal process). Hence, the difference between $q_{2}, q_{3}$ is neg. Consider the $L_{1}$-distance between two pairs $\left(p_{0}, p_{1}\right),\left(p_{0}^{\prime}, p_{1}^{\prime}\right)$ (defined as $\left.\left|p_{0}-p_{0}^{\prime}\right|+\left|p_{1}-p_{1}^{\prime}\right|\right)$. It follows that either the distance between $q_{1}$ and $q_{2}$ is at least 1 - neg or the distance between $q_{3}$ and $q_{4}$ is at least $1-$ neg. Assume, without loss of generality, that the first is true. We now argue that, for some $i(2 \leq i<n)$, the two pairs

$$
\left(p_{0}^{i}, p_{1}^{i}\right) \triangleq q\left(B^{0}, M_{1}^{0}, \ldots, M_{i}^{0}, 0, \ldots, 0\right)
$$

and

$$
\left(p_{0}^{i-1}, p_{1}^{i-1}\right) \triangleq q\left(B^{0}, M_{1}^{0}, \ldots, M_{i-1}^{0}, 0, \ldots, 0\right)
$$

are such that either $\max \left\{p_{0}^{i}, p_{0}^{i-1}\right\}+\max \left\{p_{1}^{i}, p_{1}^{i-1}\right\}>1-$ neg $+1 /(5 n)$ or $\min \left\{p_{0}^{i}, p_{0}^{i-1}\right\}+\min \left\{p_{1}^{i}, p_{1}^{i-1}\right\}<1-\operatorname{neg}-1 /(5 n)$. Otherwise, this in particular implies that all the points $\left(p_{0}^{i}, p_{1}^{i}\right)$ are such that $1-$ neg $-1 /(5 n)<p_{0}^{i}+p_{1}^{i}<$ $1-$ neg $+1 /(r n)$. This in turn implies that the distance between two adjacent pairs is smaller than $1 /(2 n)$ and the total distance between $q_{1}$ and $q_{2}$ is less than $0.5<1-$ neg, contradicting what we know about this distance. Suppose that for some $i$ we have $\max \left\{p_{0}^{i}, p_{0}^{i-1}\right\}+\max \left\{p_{1}^{i}, p_{1}^{i-1}\right\}>1-$ neg $+1 /(5 n)$; we describe a strategy for the adversary to bias the output towards 1 (in the other case there is a dual strategy to bias the output towards 0 ). Now, the adversary picks another corrupted player $P_{j}$ : if $i>2$ the adversary uses $P_{j}=P_{i}$; otherwise, if $i=2$ the adversary uses, say, $P_{j}=P_{3}$. The adversary lets $P_{2}$ play in the first round as in $\left(B^{0}, M_{1}^{0}, \ldots, M_{i}^{0}, 0, \ldots, 0\right)$. In the second round, the adversary uses $P_{j}$ as a Trojan horse; it lets $P_{j}$ first get all the second round messages by other players (rushing) and checks which message from $P_{2}$ (either $M_{i}^{0}$ or 0 ) will cause $P_{j}$ to output 1 (the idea being that a difference which is only in the first round message sent to $P_{i}$ will only influence the second round message sent by $P_{i}$ and no other message). If there is such a message then $P_{j}$ proceeds as if it got this message (and since $P_{j}$ is honest-looking its output is the same as the output of all good players); otherwise, the adversary picks one of the two messages arbitrarily. Since $x_{1}$ is randomly chosen and by the choice of $i$, the probability of getting the output 1 is now $0.5 \cdot\left(\max \left\{p_{0}^{i}, p_{0}^{i-1}\right\}+\max \left\{p_{1}^{i}, p_{1}^{i-1}\right\}\right)$ which is significantly larger than 0.5 , as needed.

\subsection{The Function AND, or: The Advantage of Being Selfish}

In this section we consider the function $\operatorname{AND}\left(x_{1}, x_{2}, \ldots, x_{n}\right)$ defined as $x_{1} \wedge x_{2}$. We will show that this function cannot be securely computed in two rounds. This case differs from the previous ones in that the relevant impossibility result does 
not rely on the adversary's rushing capability, and thus holds also in the fully synchronous model. The intuition here is also different. In the previous examples, we showed that the adversary could violate the correctness of the protocol by inducing some invalid output distribution. In the current case, we will show that the real-life adversary can somehow gain an information advantage over its idealprocess counterpart. This will be achieved by practicing a typical selfish behavior: the adversary, corrupting $P_{2}$ and some other player $P_{j}(j>2)$, will manage to collect information about $x_{1}$ from all uncorrupted players and at the same time refuse to contribute its own share of information to the community. This will allow the real-life adversary to obtain a better prediction of the unknown input than any of the uncorrupted players, which (for the specific case of the AND function) is impossible to achieve in the ideal process.

Theorem 4. There is no 2-round (computationally) secure AND protocol, for any $t \geq 2$ and an arbitrarily large number of players $n \geq 3$, even in the fully synchronous model.

Proof. Similarly to the previous proofs, the adversary corrupts player $P_{2}$, which together with an additional player is used to violate the properties of the alleged protocol. We also follow some of the previous notation. Specifically, by $(B, M)$ we denote some joint distribution of the broadcast and the private messages (respectively) sent by $P_{2}$ in Round 1 of the protocol. By $\left(B^{b}, M^{b}\right)(b \in\{0,1\})$ we denote the honest distribution corresponding to the input $x_{2}=b$. Consider a scenario where $P_{1}$ chooses its input $x_{1}$ at random. We now argue that in the ideal process the best prediction that the adversary has for the value of $x_{1}$ is OUT, the output of the good players, whereas we show a real-life adversary that can guess $x_{1}$ with significantly better probability than by using this output. For $(B, M)$ as above, let $\operatorname{COR}(B, M)$ denote the correlation of OUT with $x_{1}$ provided that the first round messages by $P_{2}$ are distributed as in $(B, M)$. Namely,

$\operatorname{COR}(B, M) \triangleq\left|\operatorname{Pr}\left[\mathrm{OUT}=1 \mid x_{1}=1,(B, M)\right]-\operatorname{Pr}\left[\mathrm{OUT}=1 \mid x_{1}=0,(B, M)\right]\right|$.

Consider the following four quantities:

$$
\begin{aligned}
& q_{1}=\operatorname{COR}\left(B^{0}, M_{1}^{0}, M_{2}^{0}, \ldots, M_{n}^{0}\right) \\
& q_{2}=\operatorname{COR}\left(B^{0}, M_{1}^{0}, 0, \ldots, 0\right) \\
& q_{3}=\operatorname{COR}\left(B^{1}, M_{1}^{1}, \ldots, \ldots, 0\right) \\
& q_{4}=\operatorname{COR}\left(B^{1}, M_{1}^{1}, M_{2}^{1}, \ldots, M_{n}^{1}\right)
\end{aligned}
$$

By the protocol's correctness, it follows that $q_{1}=$ neg while $q_{4}=1-$ neg. In addition, similarly to Lemma 2 , the distributions $\left(B^{0}, M_{1}^{0}\right)$ and $\left(B^{1}, M_{1}^{1}\right)$ must be indistinguishable (otherwise an adversary corrupting, in a passive manner, the player $P_{1}$ gets a significantly better than $50 \%$ chance of guessing the input $x_{2}$ even when the honest players' output is 0 ; this is impossible in the ideal process). Hence, the difference between $q_{2}, q_{3}$ is neg. It follows that either the distance between $q_{1}$ and $q_{2}$ is at least 0.5 - neg or the distance between $q_{3}$ and $q_{4}$ is at least $0.5-$ neg. Assume, without loss of generality, that the first is true. Therefore, for some $i(2 \leq i<n)$ the quantity $\operatorname{COR}\left(B^{0}, M_{1}^{0}, \ldots, M_{i}^{0}, 0, \ldots, 0\right)$ is significantly smaller than $\operatorname{COR}\left(B^{0}, M_{1}^{0}, \ldots, M_{i+1}^{0}, 0, \ldots, 0\right)$ (by at least $(1-$ 
neg) $/(2 n))$. Now, the adversary picks another corrupted player $P_{j}$ : if $i>2$ the adversary uses $P_{j}=P_{i}$; otherwise, if $i=2$ the adversary uses, say, $P_{j}=P_{3}$. The adversary plays as in the distribution $\left(B^{0}, M_{1}^{0}, \ldots, M_{i}^{0}, 0, \ldots, 0\right) 9$ so as to guarantee a lower correlation between the output of good players, OUT, and $x_{1}$; however, it also uses $P_{j}$ to compute the output $\mathrm{OUT}^{\prime}$ of the good players $\left(P_{j}\right.$ behaves like such a player) in case the messages of $P_{2}$ come from the distribution $\left(B^{0}, M_{1}^{0}, \ldots, M_{i+1}^{0}, 0, \ldots, 0\right)$. This value $\mathrm{OUT}^{\prime}$ is significantly better correlated with $x_{1}$ than the actual output OUT.

Finally, we argue that in the ideal process the adversary has no better predictor for the value of $x_{1}$ than OUT. For this, simply consider all the 4 potential views $\left(x_{2}, \mathrm{OUT}\right)$ that the adversary may see. The view $(0,1)$ is impossible; for both views $(1,0)$ and $(1,1)$ we have $x_{1}=$ OUT. If the view is $(0,0)$ then the adversary has no information about $x_{1}$; in such a case, guessing the value $x_{1}=$ OUT is correct with probability $1 / 2$ and is as good as any other way of guessing. Hence, OUT is an optimal predictor for $x_{1}$.

\section{Concluding Remarks}

We have answered some of the main qualitative questions concerning the round complexity of secure multiparty computation in our standard model. In particular, we have shown that security against an active adversary requires strictly more interaction than security against a passive adversary, and that general secure computation tasks require more interaction than distributed zero-knowledge and similar tasks. As a future goal, it remains to find a characterization of secure computation tasks according to their exact round complexity. This question appears to be nontrivial, partly due to the difficulty of capturing the exact power of an adversary attacking an ideal-process implementation of complex functions.

\section{References}

1. J. Bar-Ilan and D. Beaver. Non-cryptographic fault-tolerant computing in a constant number of rounds. In Proc. 8th ACM PODC, pages 201-209. ACM, 1989.

2. D. Beaver. Minimal-Latency Secure Function Evaluation. In Eurocrypt '00, pages 335-350, 2000. LNCS No. 1807.

3. D. Beaver, J. Feigenbaum, J. Kilian, and P. Rogaway. Security with low communication overhead (extended abstract). In Proc. of CRYPTO '90.

4. D. Beaver, S. Micali, and P. Rogaway. The round complexity of secure protocols (extended abstract). In Proc. 22nd STOC, pages 503-513. ACM, 1990.

5. M. Ben-Or, S. Goldwasser, and A. Wigderson. Completeness Theorems for Noncryptographic Fault-Tolerant Distributed Computations. Proc. 20th STOC88, pp. $1-10$.

6. M. Ben-Or and N. Linial. Collective Coin-Flipping. In Randomness and Computation, pages 91-115, 1990.

${ }^{9}$ namely it samples from the distribution $\left(B^{0}, M^{0}\right)$ and creates the hybrid distribution by replacing some of the messages by 0 -messages. 
7. P. Berman, J. Garay, and K. Perry. Bit Optimal Distributed Consensus. In R. Yaeza-Bates and U. Manber, editors, Computer Science Research, pages 313322. Plenum Publishing Corporation, 1992.

8. C. Cachin, J. Camenisch, J. Kilian, and J. Muller. One-round secure computation and secure autonomous mobile agents. In Proceedings of ICALP'00, 2000.

9. R. Canetti. Security and composition of multiparty cryptographic protocols. Journal of Cryptology, 13(1):143-202, 2000.

10. D. Chaum, C. Crepeau, and I. Damgard. Multiparty Unconditionally Secure Protocols. In Proc. 20th STOC88, pages 11-19.

11. R. Cramer and I. Damgård. Secure distributed linear algebra in a constant number of rounds. In Proc. Crypto 2001.

12. R. Cramer, I. Damgård, S. Dziembowski, M. Hirt, and T. Rabin. Efficient multiparty computations with dishonest minority. In Eurocrypt '99, pages 311-326, 1999. LNCS No. 1592.

13. Uri Feige, Joe Kilian, and Moni Naor. A minimal model for secure computation (extended abstract). In Proc. 26th STOC, pages 554-563. ACM, 1994.

14. P. Feldman and S. Micali. An Optimal Algorithm for Synchronous Byzantine Agreement. SIAM. J. Computing, 26(2):873-933, 1997.

15. F. Gavril. Manuscript, 1974.

16. R. Gennaro, S. Halevi, and T. Rabin. Round-optimal zero knowledge with distributed verifiers. www.research.ibm.com/security, 2002.

17. R. Gennaro, Y. Ishai, E. Kushilevitz, and T. Rabin. The Round Complexity of Verifiable Secret Sharing and Secure Multicast. In Proc. 33th STOC. ACM, 2001.

18. Rosario Gennaro. Achieving Independence Efficiently and Securely. In Proc. 14th ACM PODC, pages 130-136. ACM, 1995.

19. O. Goldreich. Secure multi-party computation (manuscript). www.wisdom.weizmann.ac.il/ oded/pp.html, 1998.

20. O. Goldreich. Foundation of Cryptography - Fragments of a Book. ECCC 1995. Available online from http://www.eccc.uni-trier.de/eccc/.

21. O. Goldreich, S. Micali, and A. Wigderson. How to Play Any Mental Game. In Proc. 19th STOC, pages 218-229. ACM, 1987.

22. Y. Ishai and E. Kushilevitz. Private simultaneous messages protocols with applications. In ISTCS97, pages 174-184, 1997.

23. Y. Ishai and E. Kushilevitz. Randomizing polynomials: A new representation with applications to round-efficient secure computation. In Proc. 41st FOCS, 2000.

24. Y. Ishai and E. Kushilevitz. Perfect Constant-Round Secure Computation via Perfect Randomizing Polynomials. In Proc. ICALP '02.

25. L. Lamport, R.E. Shostack, and M. Pease. The Byzantine generals problem. ACM Trans. Prog. Lang. and Systems, 4(3):382-401, 1982.

26. Y. Lindell. Parallel Coin-Tossing and Constant-Round Secure Two-Party Computation. In Crypto '01, pages 171-189, 2001. LNCS No. 2139.

27. N. Lynch. Distributed Algorithms. Morgan Kaufman, 1996.

28. R. Ostrovsky and M. Yung. How to withstand mobile virus attacks. In Proc. 10th ACM PODC, pages 51-59. ACM, 1991.

29. M. Pease, R. Shostak, and L. Lamport. Reaching Agreement in the Presence of Faults. Journal of the ACM, 27(2):228-234, 1980.

30. T. Rabin and M. Ben-Or. Verifiable Secret Sharing and Multiparty Protocols with Honest Majority. In Proc. 21st STOC, pages 73-85. ACM, 1989.

31. T. Sander, A. Young, and M. Yung. Non-Interactive CryptoComputing For NC1. In Proc. 40th FOCS, pages 554-567. IEEE, 1999.

32. A. C-C. Yao. How to Generate and Exchange Secrets. In Proc. 27th FOCS, pages 162-167. IEEE, 1986. 